\title{
Emerging flux tubes from the solar interior into the atmosphere: effects of non-constant twist
}

\author{
M. J. Murray ${ }^{1,2}$ and A. W. Hood ${ }^{1}$ \\ 1 School of Mathematics and Statistics, University of St. Andrews, North Haugh, St. Andrews, Fife, KY16 9SS, UK \\ e-mail: mjmamssl.ucl.ac.uk \\ 2 University College London, Mullard Space Science Laboratory, Holmbury St Mary, Dorking, Surrey, RH5 6NT, UK
}

Received 15 October 2007 / Accepted 7 December 2007

\begin{abstract}
Context. Observations of large-scale solar emergence events indicate that the magnetic field is already twisted prior to its emergence. However, the nature of twist of the pre-emergence field is largely unknown.

Aims. By testing two different twist profiles for subsurface magnetic flux tubes, we aim to identify any differences in the emergence process of the field and its atmospheric signatures that occur as a result of the twist. Given that differences do occur, future comparisons of these emergence results with observations should reveal specific properties of the twist of the subsurface magnetic field.

Methods. Using a 3D numerical MHD code, we consider a simple stratified model, comprising of one solar interior layer and three overlying atmospheric layers. We set a horizontal, twisted flux tube in the lowest layer and prescribe the central protion along the tube's length to be buoyant so that it will rise towards the surface. We test two different non-constant twist profiles for the tube and perform a parameter study for each profile, looking for differences and similarities during the rise, emergence and expansion stages of the tube's evolution.

Results. We find that, irrespective of the tube's twist profile, if the tube initially has a low tension force then it will experience greater expansion and consequential weakening of its field strength during the rise through the solar interior. Thus, upon reaching the solar surface it will fail to undergo a magnetic buoyancy instability and will not emerge into the atmosphere. For those tubes that do emerge into the atmosphere, there is little distinction between the atmospheric field and few indicators as to the initial twist profile of the tube. In general, tubes with stronger tension forces have a faster growth rate of the magnetic buoyancy instability, while tubes with weaker tension forces expand to a greater degree in the horizontal direction post-emergence. Synthesised magnetograms at the solar surface do vary between the two tested twist profiles but only in cases with initially low tension forces.

Conclusions. Upon emergence, it appears that most of the specific details of the tube's initial twist are lost. The field must initially be sufficiently twisted such that it is able to undergo a magnetic buoyancy instability but such a level of twist subsequently results in the emerging flux having generic atmospheric characteristics. Only in cases with an initially low tension force is it possible to make some distiction post-emergence by considering magnetograms at the solar surface.
\end{abstract}

Key words. magnetohydrodynamics (MHD) - Sun: magnetic fields - Sun: interior - Sun: atmosphere - methods: numerical

\section{Introduction}

Sunpots have long been observed and their general development is well documented (for a detailed discussion see Solanki 2003, and references therein). The growth of a pair of sunspots begins with the emergence of individual arched strands of flux or loops in a confined area (Zwaan 1985). Over time, the distance between the footpoints of each loop increases as the loop rises higher into the atmosphere, with the direction of the footpoint separation being similar for all of the individual loops. The footpoints appear to collect in two distinct regions that develop into dark pores and, subsequently, into a pair of sunspots. For such large amounts of flux to be emerging in a concentrated region, it seems plausible to assume that the flux resides in some accummulated state before emerging. Thus, it is taken to be fact that sunspots mark the location where a section of a large subsurface bundle of flux has erupted through the surface, leaving its footpoints rooted in the solar interior.

In addition, it is widely believed that the magnetic field of the subsurface flux bundle is twisted and intertwined, prior to emergence. The observational evidence in support of the theory that flux ropes exist in a sheared state pre-emergence has developed substantially since it was first proposed (Babcock 1961;
Piddington 1975). Firstly, the orientation of newly emerging field changes with respect to the polarity inversion line of the active region (Frazier 1972; Lites et al. 1995; Schmieder et al. 1996). The first loops to emerge are perpendicular to this line, while the orientation of successively emerging loops appears to rotate such that the final loops are practical parallel to the inversion line. The rate at which the loops rotate is far greater than typical photospheric motions of $\sim 0.5 \mathrm{~km} \mathrm{~s}^{-1}$ (Lites et al. 1995) and flows around sunspots are radially outwards over the moat rather than rotational (Piddington 1981). Secondly, there is no significant delay between the emergence of the new field and the detection of currents in the photosphere (Leka et al. 1996). Thus, the current must be associated with the field before it emerges since motions in the photosphere would take too long to tangle the field and generate the current. Thirdly, comparisons of $\mathrm{H} \alpha$ and X-ray structures with potential (Leka et al. 1996) and linear force-free (Schmieder et al. 1996) field extrapolations do not agree, indicating that the emerging field is in a non-potential state. All of these points strongly support a model in which the field of the rope is twisted prior to its arrival in the photosphere.

To date, there have been a limited number of observational studies carried out that aim to determine the level of twist 
associated with large-scale emerging flux. Assuming the field is force-free, these studies use vector magnetogram data to determine the value of a twist parameter, $\alpha_{\mathrm{p}}$, by satisfying $\nabla \times \boldsymbol{B}=$ $\alpha_{\mathrm{p}} \boldsymbol{B}$ (see Canfield et al. 1993 for calculation details). The results reveal that each hemisphere has an associated dominance in the sign of $\alpha_{p}$ of emerging active regions (Pevtsov et al. 1995). Estimates of the value of $\alpha_{\mathrm{p}}$ are reported to be $\mathrm{O}\left(10^{-2} \mathrm{Mm}^{-1}\right)$ (Green et al. 2002; Burnette et al. 2004).

Previous flux emergence simulations have considered horizontal flux sheets with uniformly directed magnetic fields (Kaisig et al. 1990; Shibata et al. 1992; Matsumoto et al. 1993; Yokoyama \& Shibata 1995; Miyagoshi \& Yokoyama 2003; Isobe et al. 2005) and twisted flux tubes as the seed magnetic field. Of these, only the simulations that use a flux tube as the seed magnetic field have been able to create variation in the orientation of the field as further flux emerges. However, the majority of the simulations using flux tubes for emergence consider a constant twist flux tube for simplicity and to aid comparison between experiments (Magara 2001, 2004, 2006; Magara \& Longcope 2001, 2003; Fan 2001; Archontis et al. 2004, 2005, 2006, 2007; Manchester et al. 2004; Galsgaard et al. 2005, 2007; Leake \& Arber 2006; Murray et al. 2006; Murray \& Hood 2007). Prescribing constant twist yields a flux tube whose fieldlines rotate about the axis through the same angle over the same set length in the axial direction, irrespective of radius.

The simulations that use non-constant twist flux tubes transport the flux from the solar interior into the atmosphere kinematically and are concerned only with the subsequent development of the coronal magnetic field (Fan \& Gibson 2003, 2004; Fan 2005; Gibson \& Fan 2006). Thus, to date, there have been no self-consistent models of flux emergence where the flux tube has not been initially prescribed with constant twist. There is no reason to assume that flux tubes formed in the solar interior should have constant twist and, therefore, in this paper we will investigate whether changing the twist of the tube to be non-constant modifies the emergence process.

The degree of twist associated with the magnetic field of a flux tube is a key parameter in deterimining the tube's overall evolution. The level of twist is directly related to the magnitude of the tube's tension force, which is responsible for maintaining the cohesion of the tube. Previous studies have shown that as a flux tube with low twist rises through the solar interior it will suffer from distortion (Moreno-Insertis \& Emonet 1996; Emonet \& Moreno-Insertis 1998). Hughes et al. (1998) have carried out a study of constant twist flux tubes rising through the solar interior in comparison with non-constant twist tubes. They found that, in the latter case, the maximum strength of the tube's transverse field needed to prevent distortion was smaller. For the levels of twist we will be considering, we do not expect to find the distortion described by Hughes et al. (1998), which results in the tube developing side lobe structures. Finally, emergence simulations of constant twist tubes have found that the flux will not be susceptible to the buoyancy instability upon entering the photosphere if the level of twist is initially too low (Murray et al. 2006). In the case of non-constant twist flux tubes, we believe that there will also be a minimum level of twist required to ensure emergence.

We will be testing two different flux tube profiles that give decreasing and increasing twist with radius. For each of these profiles, we will also perform a parameter study in order to understand the importance of the rate of change of the twist with radius.

The structure of this paper is as follows: Sect. 2 will introduce the numerical code used for the simulations and the setup of the domain model. In Sect. 3, we will present the results from the simulations, highlighting the similarities and differences of the tube's evolution that are consequences of the twist profiles and their associated parameter spaces. Finally, in Sect. 4 we will summarise our findings and consider whether observational studies can use our results to determine the exact twist of the subsurface pre-emergence field.

\section{Model setup}

\subsection{Equations and numerical code}

For the experiments in this paper, we solve the threedimensional, time-dependent, resistive MHD equations numerically. These equations are:

$$
\begin{aligned}
\frac{\partial \rho}{\partial t} & =-\nabla \cdot(\rho \boldsymbol{v}), \\
\frac{\partial(\rho \boldsymbol{v})}{\partial t} & =-\nabla \cdot(\rho \boldsymbol{v} \boldsymbol{v}+\hat{\tau})-\nabla p+\rho \boldsymbol{g}+\boldsymbol{J} \times \boldsymbol{B}, \\
\frac{\partial e}{\partial t} & =-\nabla \cdot(e \boldsymbol{v})-p \nabla \cdot \boldsymbol{v}+Q_{\text {Joule }}+Q_{\mathrm{visc}}, \\
\frac{\partial \boldsymbol{B}}{\partial t} & =-\nabla \times \boldsymbol{E}, \\
\boldsymbol{E} & =-\boldsymbol{v} \times \boldsymbol{B}+\frac{\boldsymbol{J}}{\sigma}, \\
\boldsymbol{J} & =\nabla \times \frac{\boldsymbol{B}}{\mu}, \\
p & =\rho T \frac{\mathcal{R}}{\tilde{\mu}},
\end{aligned}
$$

with density $\rho$, velocity $\boldsymbol{v}$, viscous stress tensor $\hat{\tau}$, gas pressure $p$, acceleration due to gravity $\boldsymbol{g}$, electric current density $\boldsymbol{J}$, magnetic field $\boldsymbol{B}$, thermal energy per unit volume $e=p /(\gamma-1)$, Joule dissipation $Q_{\text {Joule }}$, viscous dissipation $Q_{\text {visc }}$, electrical resistivity $\sigma$, magnetic permeability $\mu$, temperature $T$, gas constant $\mathcal{R}$, average atomic mass per particle $\tilde{\mu}$, respectively. $\gamma$ is the ratio of specific heats and is taken to be $5 / 3$.

We use the three-dimensional code developed by Nordlund \& Galsgaard (1997), which solves the MHD equations on staggered grids using a sixth order accurate method to evaluate spatial derivatives and a fifth order interpolation method for shifting variables between the different grids. An explicit third order predictor-corrector method is used to advance the solution in time.

A grid of $148 \times 160 \times 218$ points is used to model an integration box with a dimensionless domain $(-60,60) \times(-70,70) \times$ $(-22,70)$ in the $(x, y, z)$ coordinate system. This corresponds to a domain of $20.4 \mathrm{Mm} \times 23.8 \mathrm{Mm} \times 15.6 \mathrm{Mm}$ on the Sun. The grid has uniform spacing in the horizontal plane but is stretched in the vertical direction. The numerical resolution is highest over the region from just below the initial location of the flux tube to just above the transition region. The gridspacing represents a physical distance of $4.77 \times 10^{4} \mathrm{~m}$ at its smallest and $2.49 \times 10^{5} \mathrm{~m}$ at its largest.

The boundaries of the box are periodic in the horizontal directions and closed on the top and bottom. A damping region is included at both the top and bottom of the box to limit the reflection of waves there.

\subsection{Hydrostatic atmosphere}

The background stratification of the domain models four distinct regions of the Sun, which are initially in hydrostatic equilibrium. 
In the base of the non-dimensional domain, $-22 \leq z \leq 0$, resides the adiabatic solar interior, with a linearly increasing temperature profile with depth such that it is marginally stable to the onset of convection. Above the solar interior lie two isothermal regions. The lower isothermal region, $0 \leq z \leq 10$, represents the photosphere and the upper isothermal region, $20 \leq z \leq 70$, represents the corona. The corona is chosen to be 150 times hotter than the photosphere. Hence, in the intermediary region, $10 \leq z \leq 20$, there is a rapid increase in temperature to ensure the temperature profile is continuous across adjacent regions. This region characterised by a rapid temperature increase is termed the transition region.

We choose the non-dimensional temperature, density, and pressure scale-height to be $T_{\mathrm{ph}}=1, \rho_{\mathrm{ph}}=1, H_{\mathrm{ph}}=1$, respectively, at the base of the photosphere. Physical units are obtained by multiplying the dimensionless quantities by $T_{\mathrm{ph}}=$ $5.6 \times 10^{3} \mathrm{~K}, \rho_{\mathrm{ph}}=3 \times 10^{-4} \mathrm{~kg} \mathrm{~m}^{-3}, H_{\mathrm{ph}}=1.7 \times 10^{5} \mathrm{~m}$. From these values we define a photospheric gas pressure unit $p_{\mathrm{ph}}=1.4 \times 10^{11} \mathrm{erg} \mathrm{m}^{-3}$, a velocity unit $V \equiv\left(p_{\mathrm{ph}} / \rho_{\mathrm{ph}}\right)^{1 / 2}=$ $6.8 \times 10^{3} \mathrm{~m} \mathrm{~s}^{-1}$, a time unit $t_{\mathrm{ph}}=25 \mathrm{~s}$ and a magnetic field unit $B_{\mathrm{ph}}=1.3 \times 10^{3}$ Gauss. Surface gravity is $2.7 \times 10^{2} \mathrm{~m} \mathrm{~s}^{-1}$ and $\tilde{\mu}=1$.

\subsection{Magnetic flux tube}

In the cylindrical coordinate system, $(r, \theta, y)$, we define the magnetic field of the flux tube as $\boldsymbol{B}=\left(B_{r}, B_{\theta}, B_{y}\right)$, where

$B_{r}=0$,
$B_{\theta}=\alpha r B_{y}$,
$B_{y}=B_{0} \mathrm{e}^{-r^{2} / R^{2}}$.

We set $B_{0}=3.0$, for the maximum axial field strength, and $R=2.5$, for the radius of the tube at which the field strength has decreased to $37 \%$ of its axial value. We will vary $\alpha$ over nine experiments but the axial component of the tube's magnetic field, $B_{y}$, will be the same in all of the simulations at $t=0$.

The twist of the flux tube is given by

$\Phi=\frac{B_{\theta}}{r B_{y}}=\alpha$.

In order to create a non-constant twist profile with radius, $\alpha$ must be a function of $r$. We will investigate two different profiles given by the functions $\alpha_{1}$ and $\alpha_{2}$, where

$\alpha_{1}(r)=c \exp \left(-r^{2} / k_{1}^{2}\right)$,

$\alpha_{2}(r)=c\left(1-\exp \left(-r^{2} / k_{2}^{2}\right)\right)$.

$\alpha_{1}$ and $\alpha_{2}$ give twist profiles that decrease and increase with radius, respectively. We choose $c=0.5$ and will be investigating the parameter spaces of $k_{1}$ and $k_{2}$.

The values of $k_{1}$ and $k_{2}$ determine the rate of change of the twist with radius. As $k_{1} \rightarrow \infty$ and $k_{2} \rightarrow \infty$, we retrieve the constant twist profiles with $\alpha_{1}=0.5$ and $\alpha_{2}=0.0$ respectively. On the other hand, as $k_{1} \rightarrow 0$ and $k_{2} \rightarrow 0, \delta$ functions develop at the axis that give $\alpha_{1}=0.5$ and $\alpha_{2}=0.0$ there and $\alpha_{1}=0.0$ and $\alpha_{2}=0.5$ everywhere else.

Table 1 summaries the values of $k_{1}$ and $k_{2}$ under consideration and Fig. 1 illustrates the twist profiles of $\alpha_{1}$ and $\alpha_{2}$ for these values of $k_{1}$ and $k_{2}$.

In the case of $\alpha_{1}$, the values of $k_{1}=1.75,2.5$ result in the fieldlines at the periphery of the tube being untwisted, while all values of $k_{2}$ for $\alpha_{2}$ result in the fieldlines around the axis being
Table 1. Summary of the $k_{1}$ and $k_{2}$ parameter spaces under investigation and information regarding the line used to represent each in the figures of this paper.

\begin{tabular}{lll}
\hline \hline$k_{1}$ & & $k_{2}$ \\
\hline 1.75 & (blue solid) & 1.0 (red solid) \\
2.5 & (blue dashed) & 1.75 (red dashed) \\
5.0 & (blue dot-dashed) & 2.5 (red dot-dashed) \\
10.0 & (blue triple dot-dashed) & $5.0 \quad$ (red triple dot-dashed) \\
10000.0 (black solid) & & \\
\hline
\end{tabular}

untwisted. Untwisted fieldlines allow for the distortion of the tube as it rises through the solar interior and the severity of the distortion in these cases will be discussed in Sect. 3.1. It can be seen that a value of 10000.0 for $k_{1}$ is sufficiently large to yield a constant twist profile for $\alpha_{1}$. We will use this case as the common case against which to make comparisons between the two twist profiles.

Although the twist profiles are sufficiently different to each other, the $B_{\theta}$ component of the magnetic field is relatively similar. By considering (9), we can see that this similarity is due to the scaling with $r$, which gives $B_{\theta}=0$ at the axis, and the Gaussian $B_{y}$ component, which gives a smooth decrease to $B_{\theta}=0$ at the edge of the tube.

The tension force of a magnetic field is given by $(\boldsymbol{B} \cdot \nabla) \boldsymbol{B} / \mu$. Using (8)-(10), we find that the tension force of the tube acts radially inwards with magnitude $B_{\theta}^{2} / \mu r$. This is dependent on the form of $\alpha$, as shown in Fig. 2. However, in both the $k_{1}$ and $k_{2}$ parameter spaces we can divide the cases into two categories, namely low and high tension. We note that for increasing values of $k_{1}$ and decreasing values of $k_{2}$, the flux tubes have increasingly strong tension forces. For example, the lower tension cases are given by $k_{1}=1.75,2.5$ and $k_{2}=2.5,5.0$. If the tension is sufficiently low in these cases then, from the results of previous studies, we would expect to see some distortion to the shape of the tube during its rise through the solar interior and, upon reaching the photosphere, the flux may fail to initiate a magnetic buoyancy instability.

When discussing the results from the simulations, in addition to considering the two profiles individually, we will divide the simulations generally into cases with an initially low or high tension force, as specified above. In this way, we hope to be able to identify the individual properties of the tube's magnetic field that are important for emergence rather than specific to the individual twist profiles. When referring to the high and low tension cases, the word "initially" will be omitted but it is implied unless otherwise stated.

The axis of the flux tube is initially at a height of $z=-10$, positioning it within the solar interior. We set the tube to be in radial force balance with the surrounding medium at $t=0$ in order to prevent a sudden radial expansion or compression of the tube once the simulation begin. Additionally, we choose the central portion of the tube, at $y=0$, to be in thermal equilibrium with the environment but decrease the temperature in the tube away from this region. This variation in temperature results in the central portion of the tube being the most buoyant, with decreasing buoyancy towards the ends of the tube. The total buoyant length of the tube in the axial direction is $\sim 100$ units. The differential buoyancy will aid the development of an $\Omega$-shaped loop along the tube's axial length when the simulation starts. 

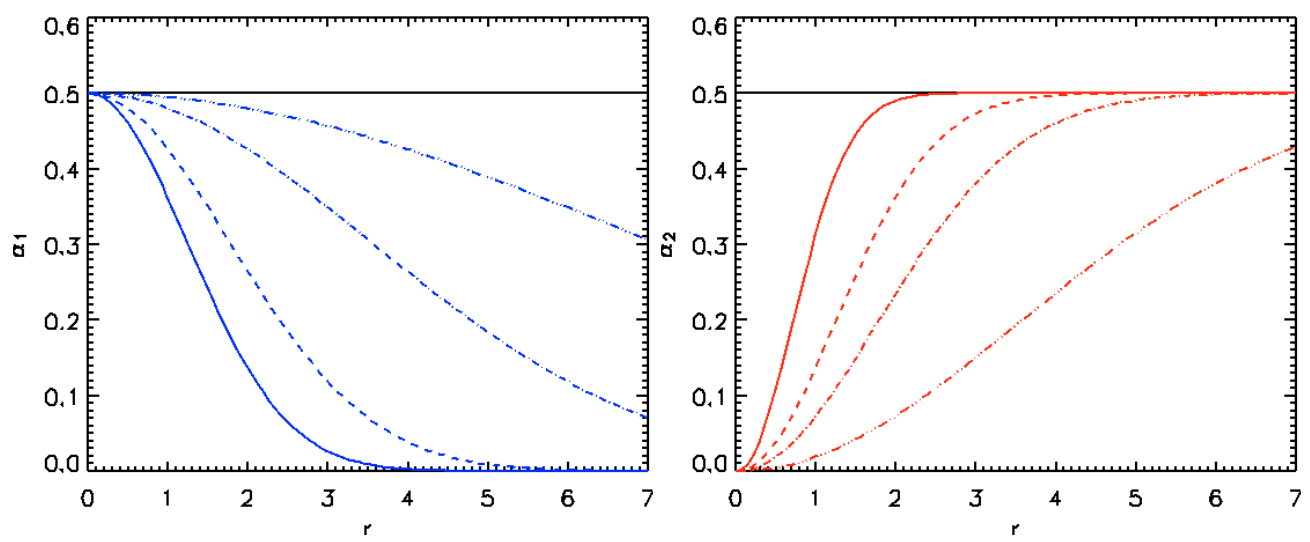

Fig. 1. Non-constant twist profiles, (left) $\alpha_{1}$ and (right) $\alpha_{2}$, as functions of radius for the values of $k_{1}$ and $k_{2}$ listed
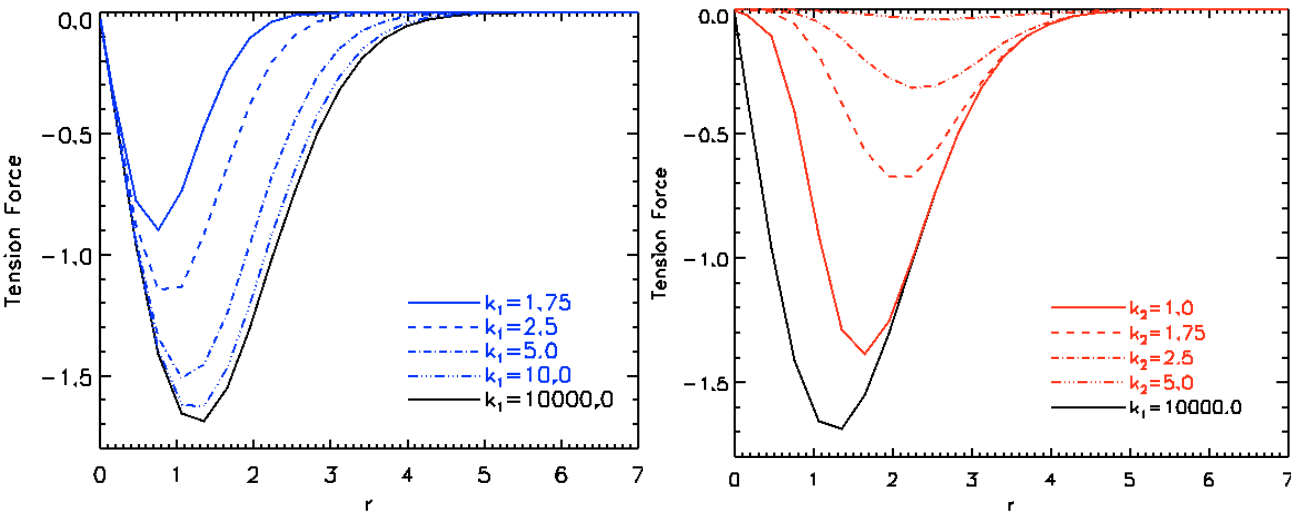

in Table 1.

Fig. 2. Tension force as a function of radius at $t=0$ for (left) $\alpha_{1}$ cases and (right) $\alpha_{2}$ cases.
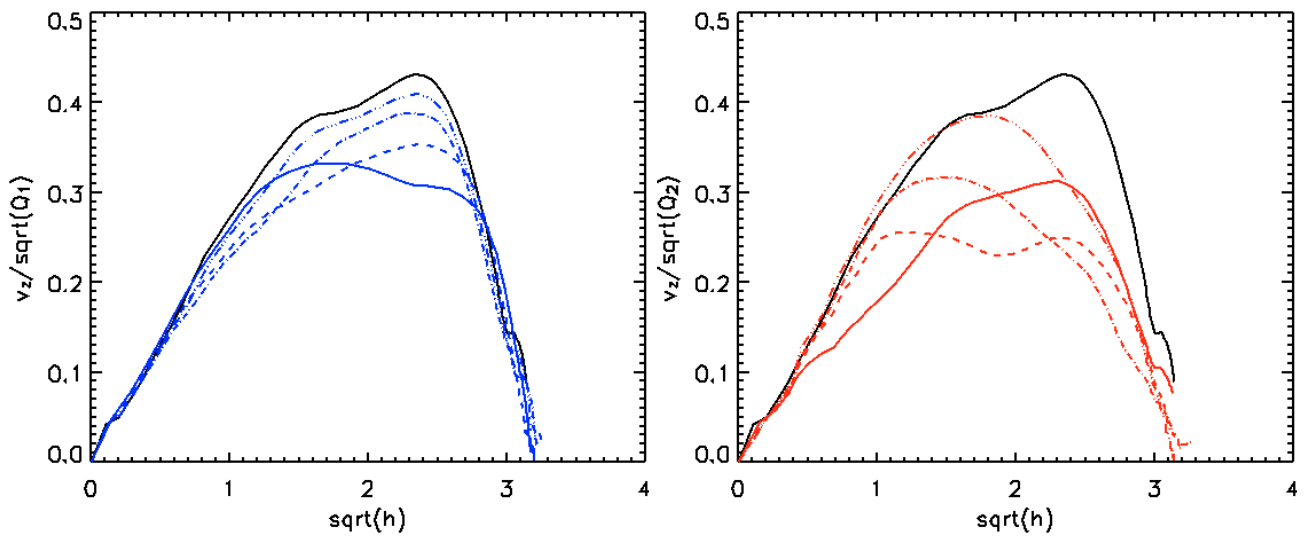

Fig. 3. Scaled axial vertical velocity plotted against the square root of the distance travelled, $h$, measured at $(x=0, y=0)$ for (left) $\alpha_{1}$ cases and (right) $\alpha_{2}$ cases.

\section{Results}

\subsection{Rise towards the solar surface}

At the start of the experiments, the only force acting on the tubes is a buoyancy force in the positive vertical direction. The tubes with a lower tension force have a lower density at their centre in order to accommodate a lower gas pressure. Thus, these tubes are initially more buoyant. Given that an explicit expression for the initial buoyancy force is known, it should be possible to scale the vertical velocity of the tube by this force and retrieve a profile that can then be used to predict the vertical velocity for any value of $k_{1}$ or $k_{2}$ for $\alpha_{1}$ or $\alpha_{2}$ respectively. A similar approach has been used successfuly by Murray et al. (2006) when changing the tube's field strength parameter, $B_{0}$.

Rather than considering the whole tube, we will consider the axis of the tube at $(x=0, y=0)$ since this is a well defined point. At the axis, $r=0$, the buoyancy force is proportional to

$Q_{1}=\frac{c^{2} d_{1}^{2}}{2}-1$,
$Q_{2}=c^{2}\left(\frac{R^{2}}{2}+\frac{d_{2}^{2}}{2}-\frac{2 R^{2} d_{2}^{2}}{\left(R^{2}+d_{2}^{2}\right)}\right)-1$,

for twist profiles $\alpha_{1}$ and $\alpha_{2}$ respectively. The new constants are defined as

$d_{1}^{2}=\frac{R^{2} k_{1}^{2}}{R^{2}+k_{1}^{2}}$

$d_{2}^{2}=\frac{R^{2} k_{2}^{2}}{R^{2}+k_{2}^{2}}$.

Thus, the initial acceleration should also be proportional to $Q_{1}$ and $Q_{2}$ for cases with $\alpha_{1}$ and $\alpha_{2}$ respectively. Firstly, for the $\alpha_{1}$ cases we have

$\frac{\partial v_{z}}{\partial t} \propto Q_{1}$,

or, alternatively,

$\frac{\partial}{\partial h}\left(\frac{v_{z}^{2}}{2}\right) \propto Q_{1}$ 

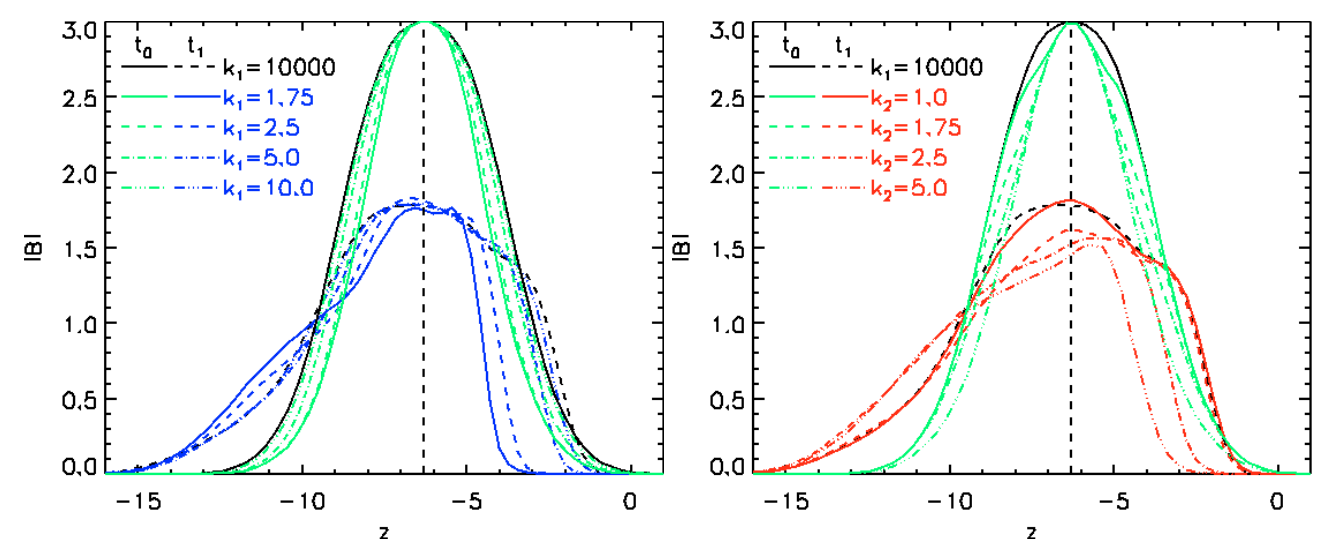

Fig. 4. Field strength, $|\boldsymbol{B}|$, as a function of height, $z$, measured at $(x=0, y=0)$, at two distinct times in each case $\left(t_{0}\right.$ and $t_{1}$ ) for (left) $\alpha_{1}$ cases and (right) $\alpha_{2}$ cases. The $t_{0}$ lines are the original profiles of the field strength at $t=0$, shifted in height such that the axis is at $z=-6.3$ (indicated by the dashed vertical line). The $t_{1}$ lines represent field strength profiles at one individually selected later time for each experiment when the axes of the tubes are at a height of $z=-6.3$. For the $\alpha_{1}$ cases, the $t_{1}$ lines represent $k_{1}=1.75,2.5,5.0,10.0,10000.0$ at $t=18,20,24,26,27$, respectively. For the $\alpha_{2}$ cases, the $t_{1}$ lines represent $k_{2}=1.0,1.75,2.5,5.0$ at $t=27,26,21,18$, respectively, and $k_{1}=10000.0$ at $t=27$.

where $h$ has units of length and measures the distance the axis has travelled from its initial height. Integrating (19) with respect to $h$ yields

$v_{z} / \sqrt{Q_{1}} \propto \sqrt{h}$,

where the constant of integration is zero. Using the same analysis for the $\alpha_{2}$ cases, we find

$v_{z} / \sqrt{Q_{2}} \propto \sqrt{h}$.

Using (20) and (21), we can now scale the vertical velocity of the axis with respect to the square root of the distance travelled by the axis. The results are shown in Fig. 3 for the $\alpha_{1}$ and $\alpha_{2}$ cases, where the axis of the tube has been identified as the point measured at $(x=0, y=0)$ where $B_{x}$ reverses sign. The above scaling is correct for the initial rise of the tube but, after rising only a short height, the scaling begins to fail. This failure occurs when the initially balanced radial Lorentz force and the gradient of the pressure deficit in the tube fall out of balance with each other. Thus, the buoyancy force is no longer the only force determining the rise of the tube. Both of the initially balanced forces reduce in size as the tube rises but, at the rear of the tube the pressure gradient decreases quicker than the Lorentz force, while at the front of the tube it decreases slower than the Lorentz force.

The differential buoyancy of the tube's plasma, in the $x-z$ plane, causes parts of the magnetic field to want to rise faster than others. In cases where the tension force is weak, we see an evacuation and accumulation of the magnetic field which distorts the tube from its initial cylindrical structure. This redistribution of field can be seen in the asymmetric field strength profiles of Fig. 4. In all cases the axis has moved away from the rear of the tube, which was less buoyant, and in the cases with a weak tension force the axis has moved towards the front of the tube, which also lacks buoyancy. In the cases with a strong tension force, the distance between the axis and the front of the tube has been approximately maintained.

In cases where the movement of the magnetic field is limited by the strong tension force, the imbalance in the radial forces remains smaller for all time. At the rear of the tube, the lack of evacuation of field maintains the gradient of the gas pressure deficit and, thus, the gradient decreases more slowly than for the cases with low tension forces. At the front of the tube, the lack of accumulation maintains the magnetic pressure gradient here and, thus, the imbalance is smaller than in the cases with low tension forces.

As the tube rises, it moves into regions where the external gas pressure and density are smaller. The reduction in the gas pressure gradient between the internal and external medium causes the tube to expand horizontally. Hence, there is a corresponding decrease in the axial component of the tube's magnetic field, $B_{y}$, so as to maintain conservation of flux. In order to evaluate the degree of expansion suffered by the different tubes, we consider the axes of the tubes as an example location. For the $\alpha_{1}$ cases, the decrease in $B_{y}$ with respect to distance travelled by the axis is independent of $k_{1}$, as shown in Fig. 5. However, for the $\alpha_{2}$ cases, we find that there is a greater decrease in $B_{y}$ with distance travelled as $k_{2}$ increases. Thus, the $\alpha_{1}$ cases experience the same degree of expansion as they rise irrespective of $k_{1}$ but the $\alpha_{2}$ cases experience a greater degree of expansion as $k_{2}$ increases.

The expansion of a tube ceases once the horizontal gas pressure gradient at the new height balances the Lorentz force. For tubes with a larger inwardly directed tension force, the degree of expansion will be smaller and, therefore, the rate of decrease in $B_{y}$ with distance travelled will also be smaller. Figure 2 shows the differing tension forces acting on the tubes at $t=0$. For the $\alpha_{1}$ cases, there is the same non-zero gradient in the tension force surrounding the axis and, thus, these tubes undergo the same degree of expansion. However, for the $\alpha_{2}$ cases there is a zero gradient in the tension force at the axis and, away from the axis, this force increases at varying rates dependent on $k_{2}$. As $k_{2}$ increases, the tension force increases more slowly with radius and, thus, there is a greater degree of expansion for the tube.

The expansion of the tube and the compression of the field at the front of the tube result in a decrease in the axial field component and an increase in the azimuthal component, respectively. This modification in the ratio of the field components causes the pitch of the tube's fieldlines to change. The pitch of a fieldline is defined as the angle, $\phi_{p}$, it makes with the axis and is given by

$\tan \left(\phi_{p}\right)=\frac{B_{\theta}}{B_{y}}$

At $t=0$, all of the tubes have a maximum pitch angle above that prescribed by Emonet \& Moreno-Insertis (1996) that is required to avoid a tube splitting apart during its rise in the solar interior.

For the $\alpha_{1}$ profile, we find that in each case the pitch in the region around the axis remains unchanged as the tubes rise through the solar interior. However, for the $\alpha_{2}$ cases, the pitch increases 

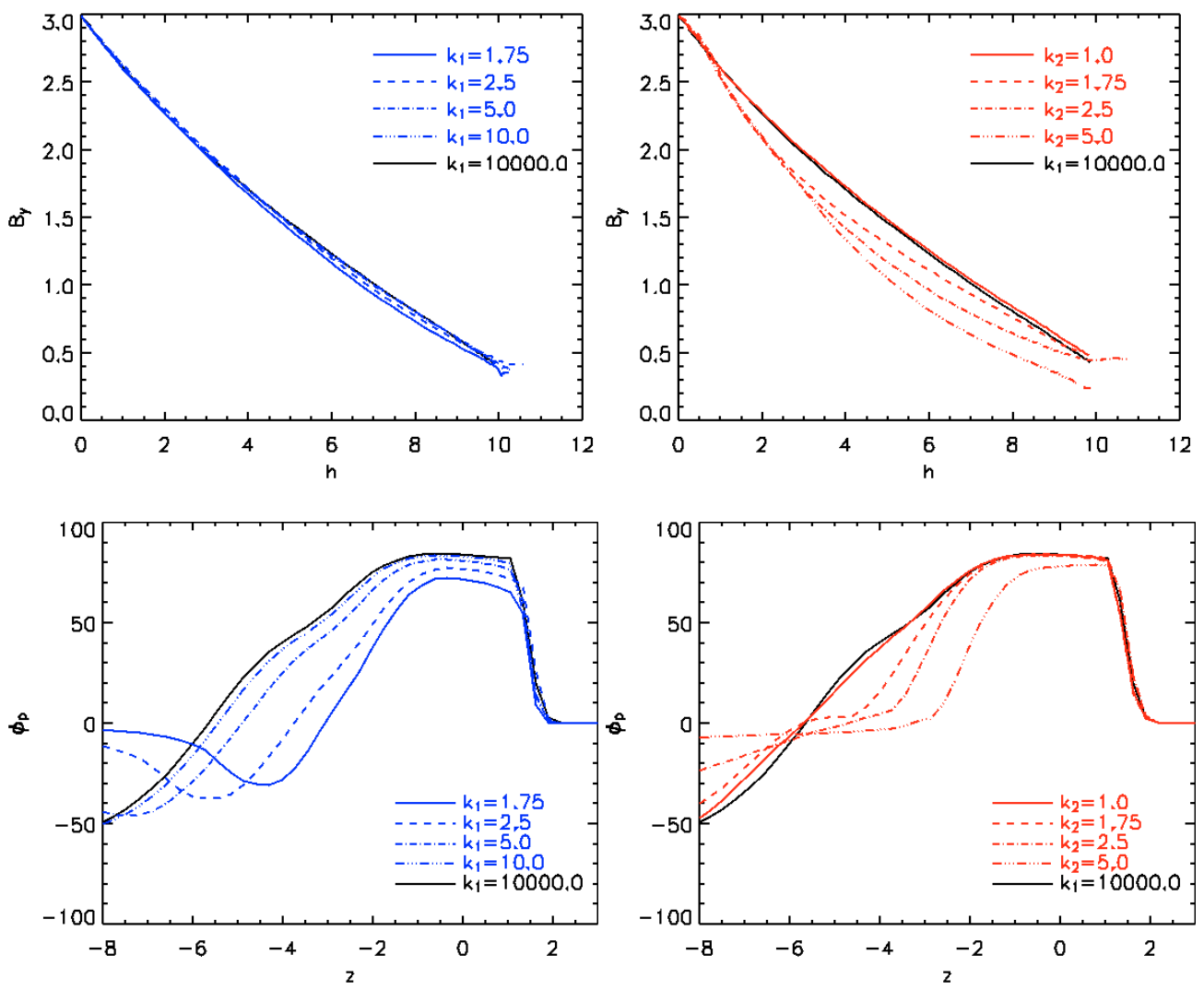

Fig. 5. Field strength at the axis, $B_{y}$, as a function of distance travelled, $h$, measured at $(x=0, y=0)$ for $(l e f t) \alpha_{1}$ cases and (right) $\alpha_{2}$ cases.

Fig. 6. Pitch of the fieldlines, $\phi_{p}$, as a function of height, $z$, at $t=30$, measured at $(x=0, y=0)$ for $(l e f t) \alpha_{1}$ cases and (right) $\alpha_{2}$ cases. in magnitude for all of the cases. These variations between the two twist profiles are as a result of the differing manner in which the axial component of the field decreases, which was discussed above. However, away from the axis, there is an increase in the pitch of the fieldlines in all of the cases due to the expansion and compression of the tube described earlier. Irrespective of the twist profile, the low tension cases, which initially have the smallest overall pitch, see the largest increase in pitch. The different increases in pitch experienced by the tubes are due to the variation in the tan profile at small and large $B_{\theta} / B_{y}$ ratios. Changes in $B_{\theta}$ or $B_{y}$ cause a much greater change in the pitch if the pitch is initially small but will have only a marginal effect when the pitch is already large.

Upon reaching the photosphere, there is little difference in the pitch profiles between the $\alpha_{1}$ and $\alpha_{2}$ cases, as shown in Fig. 6. The three $\alpha_{1}$ cases characterised by a decreasing pitch profile at outer radii have undergone two significant modifications in order to have pitch profiles that look like the other cases' profiles. Firstly, there has been an increase in pitch throughout the tubes, such that there is no longer a gentle decrease to zero pitch at the tubes' fronts but a steep gradient. This result has previously been noted by Emonet \& Moreno-Insertis (1998), who consider flux tubes rising through the solar interior, which are defined by non-constant twist profiles. Secondly, the location of the maximum pitch has migrated towards the front edge of the tube. This is inline with the work of Hughes et al. (1998), who found that after only a short rise through the solar interior, tubes with initially decreasing twist profiles actually had pitch profiles much the same as tubes prescribed with constant twist. Furthermore, they suggest that it is the maximum value of the transverse field component, $B_{\theta}$, that is important for maintaining a cohesive tube structure, free from the development of side lobes. We do not see this level of distortion here and, thus, cannot comment on whether the maximum value of the transverse field is a key parameter for cohesion.

In summary, we find that the initial vertical velocities of the tubes are well defined by the different buoyancy forces of the $\alpha_{1}$ and $\alpha_{2}$ twist profiles. However, an imbalance quickly develops between the radial pressure excess gradient and the Lorentz force of the tubes, causing the scaling of the vertical velocity with respect to the buoyancy to fail. The imbalance is largest for the cases with a low tension force due to the greater degree of evacuation and accumulation of magnetic field they experience. We see differing rates of change in the field strength of the axis for the two twist profiles. For the $\alpha_{1}$ cases, the tension force surrounding the axis is the same and, thus, the tubes experience the same amount of expansion and decrease in $B_{y}$ with distance travelled. For the $\alpha_{2}$ cases, the tension force increases more slowly with increasing $k_{2}$ and, therefore, the tubes experience different rates of expansion and decreases in the axial field component. Once the tubes reach the photosphere, their pitch profiles are relatively similar. There is no longer a smooth decrease in the pitch at outer radii for the three $\alpha_{1}$ cases with the lowest tension forces. The rate of change of the pitch with radius will be an important characteristic of the magnetic field once it expands into the atmosphere and, thus, we shall consider this in detail in Sect. 3.3.

\subsection{Emergence through the Photosphere}

As the top of the tube passes through the solar surface, it is forced to reduce its vertical velocity due to the change in stratification of the background medium. The solar interior is marginally stable to the convective instability and, therefore, when the tube became over dense compared with the surrounding it still continued rising. However, the photosphere is strongly subadiabatic and the overdense tube cannot proceed without 

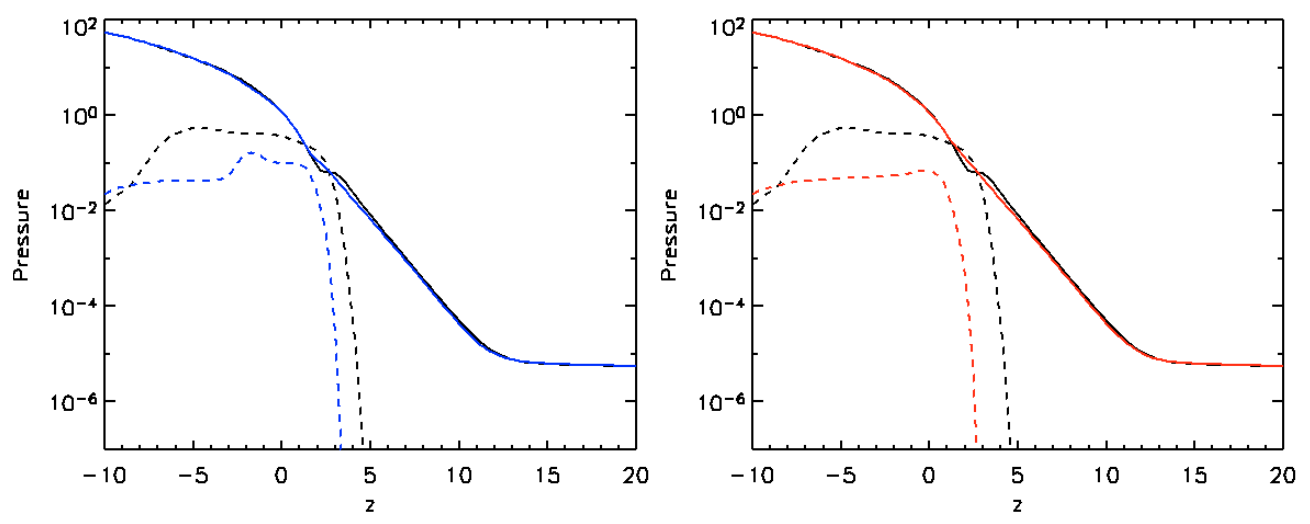

Fig. 7. Gas pressure (solid lines) and magnetic pressure (dashed lines) at $t=$ 46, measured at $(x=0, y=0)$ for $(l e f t)$ $k_{1}=10000.0$ (black) and $k_{1}=1.75$ (blue) cases and (right) $k_{1}=10000.0$ (black) and $k_{2}=5.0$ (red) cases.
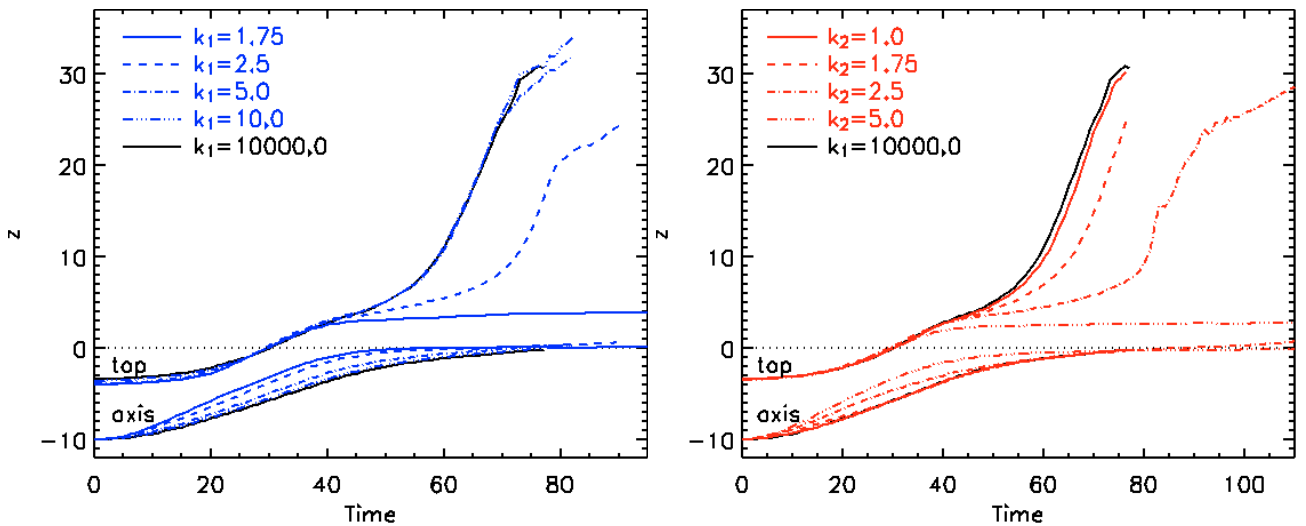

Fig. 8. Height of the top and axis of each tube over time, measured at $(x=0, y=0)$ for (left) $\alpha_{1}$ cases and (right) $\alpha_{2}$ cases. The dotted horizontal line at $z=0$ indicates the height of the solar surface.

the occurrence of a magnetic buoyancy instability (Shibata et al. 1989a,b; Matsumoto \& Shibata 1992; Kusano et al. 1998; Archontis et al. 2004; Leake \& Arber 2006; Murray et al. 2006).

We find that in the cases with a low tension force, the magnetic buoyancy instability fails to occur and flux remains trapped in the photosphere. The criterion governing the instability is (Newcomb 1961; Yu 1965; Thomas \& Nye 1975; Acheson 1979; Archontis et al. 2004; Leake \& Arber 2006; Murray et al. 2006)

$$
-H_{\mathrm{p}} \frac{\partial}{\partial z}(\log B)>-\frac{\gamma}{2} \beta \delta+{\tilde{k_{\|}}}^{2}\left(1+\frac{{\tilde{k_{\perp}}}^{2}}{{\tilde{k_{z}}}^{2}}\right),
$$

with the pressure scale-height, $H_{\mathrm{p}}$, the ratio of specific heats, $\gamma$, the plasma- $\beta$ and perturbations with wavevector $\boldsymbol{k}$ (where $\tilde{k_{\|}}$and $\tilde{k_{\perp}}$ are the horizontal components parallel and perpendicular to the magnetic field and $\tilde{k}_{z}$ is the vertical component). The superadiabatic excess, $\delta$ is given by $\delta=\nabla-\nabla_{\mathrm{ad}}$, where $\nabla$ is the actual logarithmic temperature gradient in the equilibrium stratification and $\nabla_{\text {ad }}$ is its adiabatic value.

Here, we find that as soon as the plasma- $\beta$ is less than 1 , the instability criterion becomes satisfied and flux can freely rise into the atmosphere. This is also found by Magara (2001). For all of the $\alpha_{1}$ emerging cases, we find that the instability occurs at $\sim 44$ time units but, for the $\alpha_{2}$ emerging cases, the instabilities occur over the range of 44-48 time units.

For the low tension cases, $k_{1}=1.75$ and $k_{2}=5.0$, the plasma- $\beta$ fails to fall below 1 for two reasons. Firstly, the distance between the front edges and axes of the tubes decreases quickest for these tubes due to their low tension forces and subsequent accumulation of magnetic field. Thus, at the time of these tubes' fronts entering the photosphere, their axes are at a higher location in the solar interior. Therefore, the tubes have experienced greater expansion that results in their field strengths being smaller. Secondly, these tubes fail to penetrate as high into the photosphere as the others and, therefore, are surrounded by fluid with a higher gas pressure. These points are illustrated in Fig. 7, where we have compared the magnetic pressure and gas pressure for these cases and the $k_{1}=10000.0$ emerging case.

Of the cases where the instability does occur, we find there is variation in the growth time of the instability. This is illustrated in Fig. 8 where we can see that the time between the instability criterion first being satisfied and the fastest rise phase of the top of the tube increases as $k_{1}$ decreases and $k_{2}$ increases. For long perturbation wavelengths, as is the case here, Parker (1979) states that the growth time of the instability is inversely proportional to the Alfvén speed, $V_{\mathrm{A}}=B_{0} / \sqrt{\rho}$. As shown in Fig. 9, we find that as $k_{1}$ decreases and $k_{2}$ increases, the Alfvén speed reduces as required to correspond to increasing growth times. The smaller Alfvén speed for the cases with a lower tension force is as a result of these tubes having a lower field strength at the time of the instability. As discussed above, the field strength is lower because the axes of these tubes are higher at this time and, therefore, the tubes have experienced a greater degree of expansion.

When the tubes first pass through the solar surface, they cause gravity or buoyancy waves to be generated there. The Brunt-Väisälä frequency for gravity waves, $\omega_{\mathrm{g}}$, is given by

$\omega_{\mathrm{g}}^{2}=\frac{g}{H_{0}^{*}}-\frac{g^{2}}{c_{\mathrm{s}}^{2}}$

where $g$ is gravity, $H_{0}^{*}$ is the local density scale height and $c_{\mathrm{S}}$ is the local sound speed (Roberts 1991). In our dimensionless domain, the Brunt-Väisälä frequency is $\sqrt{2 / 5}$ and, thus, we should find oscillations with a period of 9.9 time units if gravity waves are indeed being generated. By examining the vertical velocity of the flux at the front of the tubes, shown in Fig. 10, we can see that there is a clear oscillation with this period prior to the sudden increase in the vertical velocity associated with the growth 

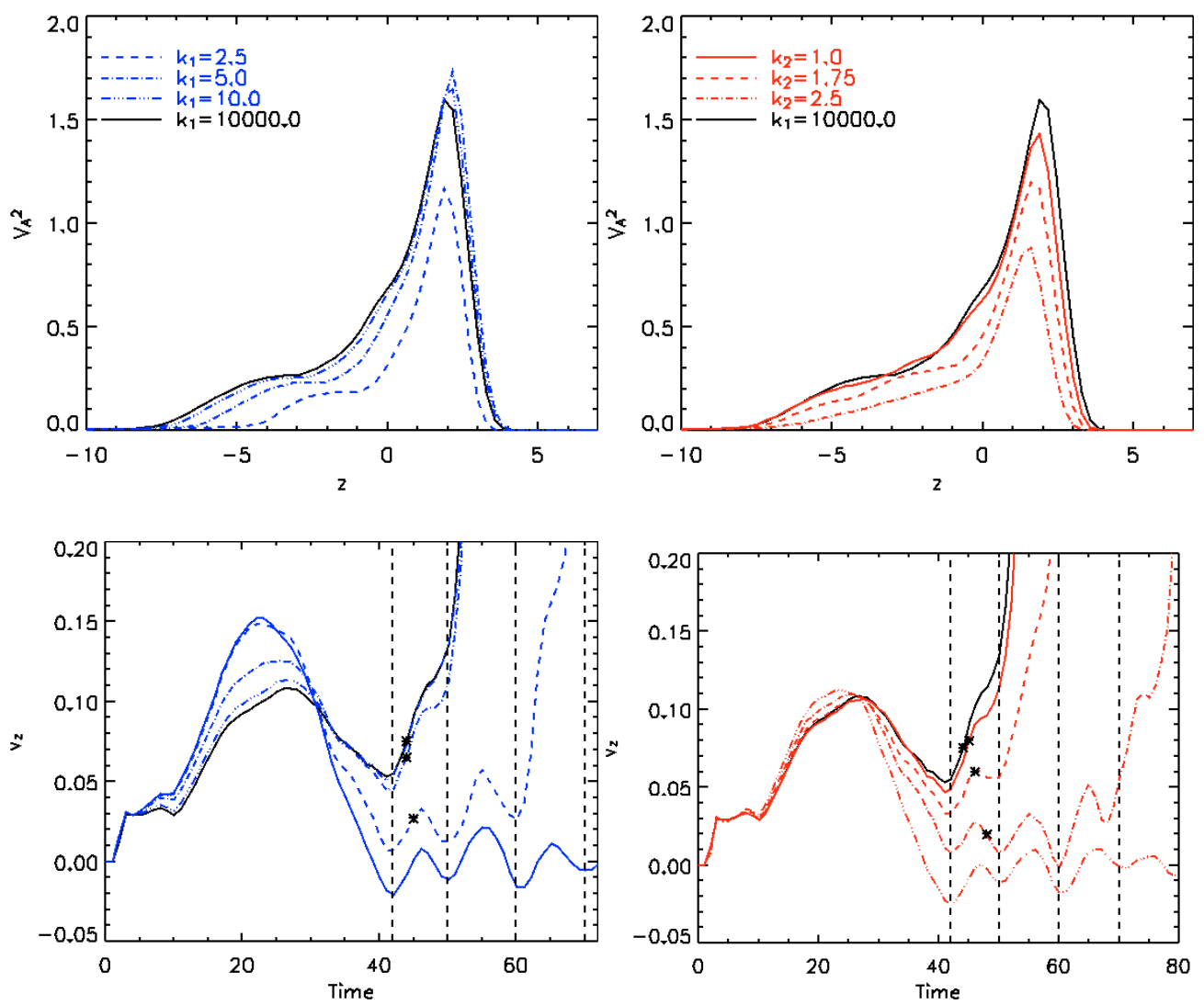

Fig. 9. Square of the Alfvén velocity as a function of height, $z$, at $t=46$, measured at $(x=0, y=0)$ for the emerging (left) $\alpha_{1}$ cases and (right) $\alpha_{2}$ cases.

Fig. 10. Vertical velocity at the front of the tube over time, measured at $(x=0, y=0)$ for (left) $\alpha_{1}$ cases and (right) $\alpha_{2}$ cases. The star on each line indicates the time at which a magnetic buoyancy instability is satisfied for each case. The instability is never satisfied for the $k_{1}=1.75$ and $k_{2}=$ 5.0 cases. The dashed vertical lines indicate the approximate troughs in the oscillating vertical velocity, which has a period of $\sim 10$ time units. This is inline with the dimensionless period expected from the Brunt-Väisälä frequency for gravity waves.
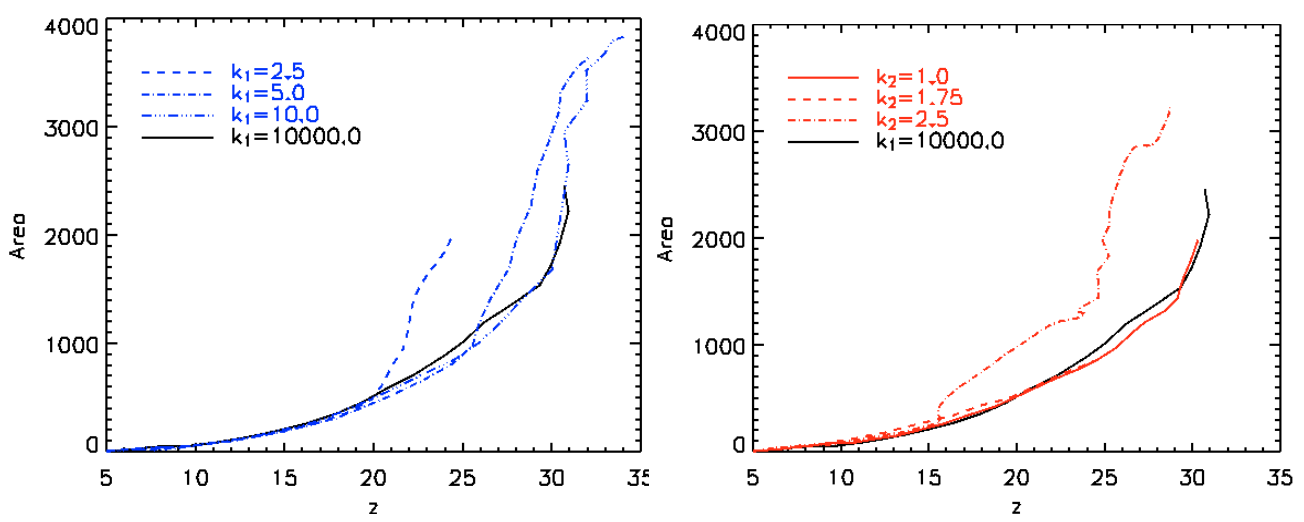

Fig. 11. Area of the $x-z$ plane at $y=0$ (for $z>5$ ) with flux passing through it as a function of the height of the top of the tube for (left) $\alpha_{1}$ cases and (right) $\alpha_{2}$ cases.

of the magnetic buoyancy instability, which eclipses the gravity waves.

In summary, during the occurrence of the buoyancy instability at the base of the photosphere, the variations in the two twist profiles seem to be of little consequence. Of more importance, is the initial tension force associated with the tubes, since the degree of distortion determines whether the buoyancy instability will occur at all and the growth rate of the instability when it does occur. For both profiles, the cases with a low tension force fail to undergo the magnetic buoyancy instability and those just above the required tension threshold initially rise more slowly into the atmosphere.

\subsection{Atmospheric expansion}

In this section, we will only be concerned with the cases that emerge. Thus, we will not be discussing the results from the $k_{1}=$ 1.75 and $k_{2}=5.0$ cases any further.
As the magnetic field of the tube advances into the atmosphere, the field undergoes rapid horizontal and vertical expansion due to the lower atmospheric gas pressure. As a measure of this expansion, we evaluate the area of the $x-z$ plane at $y=0$ (for $z>5$ ) with field passing through it. The results for the various cases are shown in Fig. 11, where the area of the plane containing flux has been plotted against the height of the top of the tube. We can see that when the tubes' tops are at the same height, the cases with lower tension forces occupy a greater area of the plane and have, therefore, experienced a greater degree of horizontal expansion. This is not surprising since a tube with a low tension force lacks the ability to constrict its expansion. The initial levels of twist and buoyant length of the tubes result in their fieldlines having a maximum of two emerged rotations, with the concave sections of the fieldlines residing below the solar surface, within the time constraints of the simulations.

Figure 12 shows the amount of flux measured in the same $x-z$ plane as a function of time. We can see that the time at which the first flux is seen in the plane increases as we move 
from the high to low tension cases. This is as a result of the slower growth rate of the buoyancy instability for the lower tension cases, which occurred when the fronts of the tubes were at a height of $z<5$ and, thus, the different rates at which the tubes' fronts advance into the atmosphere.

If all of the emerging plasma had a vertical velocity matching that of the tube front, then we could expect to see a similar increase in the gradient of the time-flux profile as we see in the vertical velocity of the tubes' fronts. However, as shown in Fig. 12, we see an approximately linear increase in all cases, indicating that the new flux entering the plane is the same at each time step but different for each case since the constant gradients are not prescribed by the same value. We find that the flux accumulates more slowly in the atmosphere for the cases with a low tension force and, therefore, flux is being transported into the atmosphere more slowly in these cases.

The different constant gradients illustrate that as each new flux layer of an individual tube satisfies the instability, it experiences the same instability growth time. Thus, at any given height, here $z=5$, a constant amount of flux will pass over the threshold with time, as found in these simulations. As a consequence of the differing growth rates between the tubes, when the tubes' fronts are at the same height, different amounts of flux have been transported into the atmosphere.

As discussed in Sect. 3.1, the pitch of the tubes' fieldlines change as the tubes rise through the solar interior, with the biggest increases seen in the $\alpha_{1}$ cases with a low tension force. However, at the time of entering the photosphere, there are still clear differences between the pitch of the fieldlines for the cases with high and low tension forces, as shown in Fig. 6. At the fronts of the tubes, there is a noticeable difference in the pitch of the emerging $\alpha_{1}$ cases but no difference in the emerging $\alpha_{2}$ cases. As we move from the high to low tension cases, there is a steeper fall in the pitch as the axis is approached from the tube front. This is as a consequence of the shorter distance between the axis and the front of the tube but, when the gradient of the pitch is measured as a function of the fractional radius, we find that the $\alpha_{1}$ cases have the same gradient and the $\alpha_{2}$ cases do not. The fractional radius is calculated as the distance between the axis and front of the tube measured at $(x=0, y=0)$ as a proportion of this distance at $t=0$.

As the tubes rise into the atmosphere, it is the field at the front of the tubes that experiences expansion. The expansion is both vertical and horizontal, which causes decreases in all of the components of the field and changes in the fieldline pitch. Figure 13 shows the fieldline pitch once the tubes' fronts have reached a certain height in the atmosphere. We find that there is little difference in the pitch of the $\alpha_{2}$ cases but a more significant difference can be seen in the pitch of the $\alpha_{1}$ cases. This would suggest that the actual pitch angle and not the gradient in the pitch of the pre-emergence field are key in determining the resulting pitch of the atmospheric field.

At the solar surface, it is possible to identify differences between the two twist profiles by constructing synthesised magnetograms, as shown in Fig. 14. At $t=76$, emergence is well under way for each of the cases depicted and, thus, in the centre of the emergence region it is fieldlines closest to the axis that are intersecting the solar surface. In the weaker tension cases, we find that a larger proportion of the emergence area contains horizontal field. For the $\alpha_{1}$ cases, the twist decreases with radius and the outer fieldlines initially have smaller pitch. Hence, the horizontal field in the magnetogram is located towards the outer edge of the emerging region. For the $\alpha_{2}$ cases, the twist increases with radius and the pitch of the fieldlines immediately surrounding

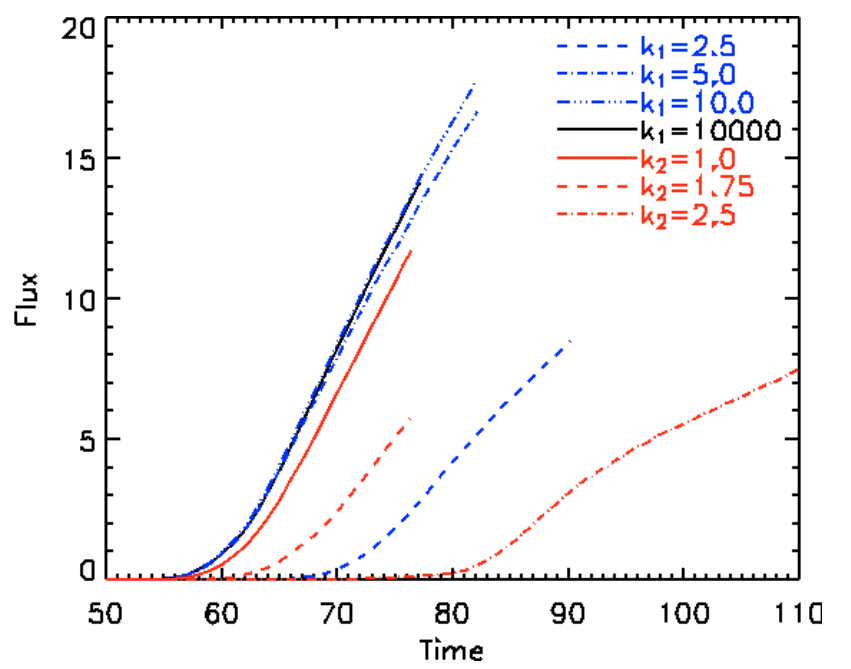

Fig. 12. Sum of the flux passing through the $x-z$ plane at $y=0$ (for $z>5$ ) plotted against time.

the axis increases more slowly than in the $\alpha_{1}$ cases. Hence, the horizontal field is situated in the centre of the emerging region in the magnetograms.

The level of twist of emerging flux is often calculated from observations by assuming the field is force-free during emergence. At the start of the simulations, the flux tubes we initialise in the solar interior are far from being force-free. This chosen property gives rise to an internal density that varies from that of the surrounding environment and results in buoyancy for the cases here. While each tube's flux is passing through the solar surface during emergence is it still not force-free, with $(\boldsymbol{J} \times \boldsymbol{B})_{z}$ taking values $\mathrm{O}\left(100 \mathrm{Gauss}^{2} \mathrm{~m}^{-1}\right)$. Hence, we do not find a meaningful value for $\alpha_{\mathrm{p}}$ at the photospheric plane, $z=0$, and the variations we find in the value of $\alpha_{\mathrm{p}}$ between the various twist profiles only indicate how far from force-free the emerging fields are. However, we do find a positive dominance in the sign of $\alpha_{p}$ that follows from the right-handed twist in the definition of the tubes.

In summary, for the cases with low tension forces, we find that the field experiences a greater degree of expansion. Although flux is transported into the atmosphere at a constant rate in each experiment, the rate of transport is slower for the tubes with low tension forces due to their associated longer instability growth time. The pitch of the fieldlines are very similar in all of the $\alpha_{2}$ cases but for the $\alpha_{1}$ cases there appears to be some difference in the pitch angle as a function of height between the high and low tension tubes. Only the synthesised magnetograms at the solar surface appear to show any distinction between the two different twist profiles but the differences appear solely for cases with low tension forces.

\section{Conclusions}

We have carried out emergence simulations of magnetic flux tubes with two different non-constant twist profiles. The $\alpha_{1}$ and $\alpha_{2}$ twist profiles are characterised by decreasing and increasing twist with radius, respectively. For each profile we considered a parameter space which varied the rate at which the twist profile increased or decreased with radius. When the twist profile increases quickly with radius towards a constant twist value of 0.5 and decreases slowly away from this value, the flux tubes have an associated high tension force. When the twist profile increases 

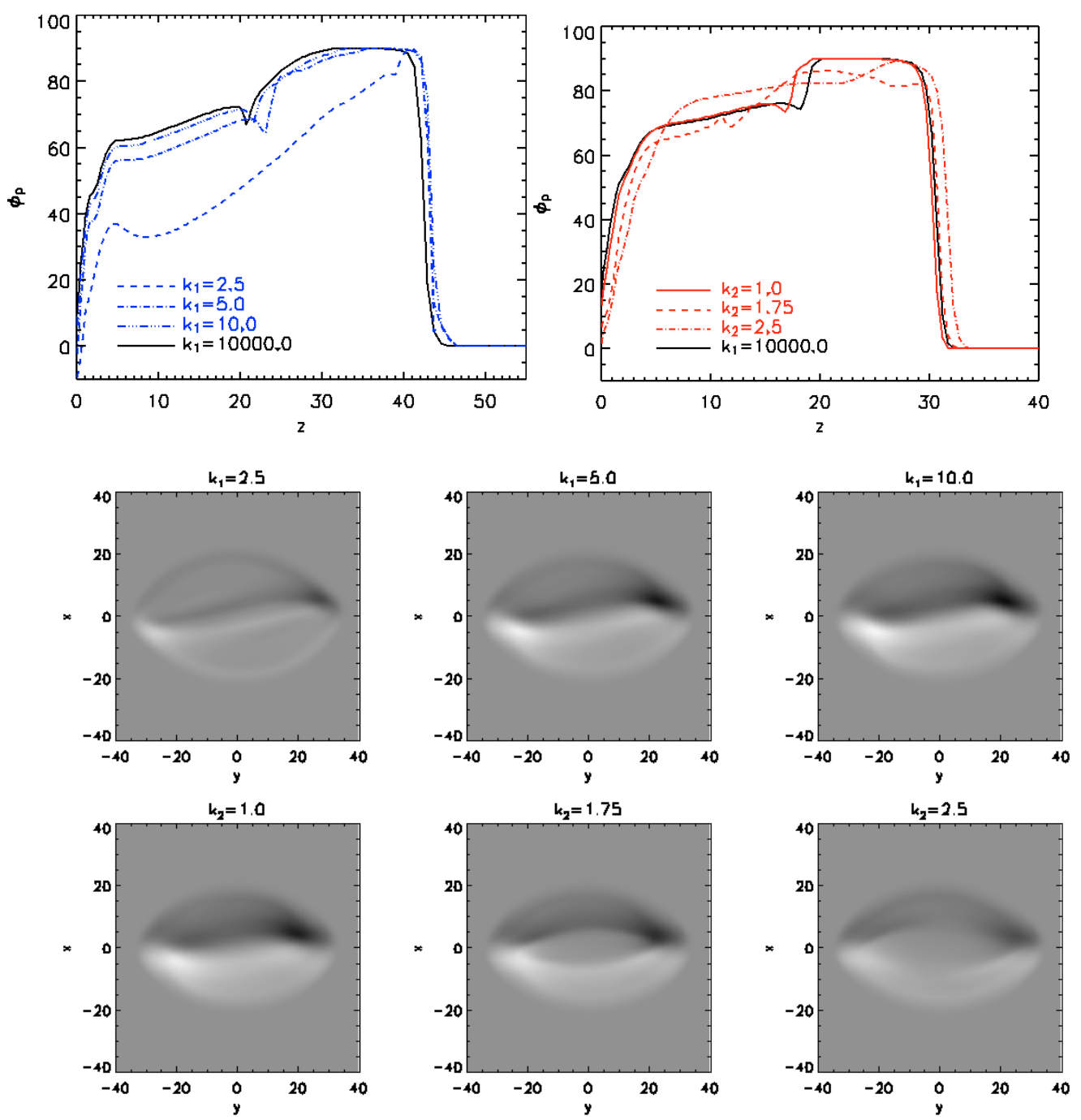

Fig. 13. Pitch of the fieldlines as a function of height, $z$, measured at $(x=0, y=0)$ for (left) $\alpha_{1}$ cases and (right) $\alpha_{2}$ cases. The times at which the lines are plotted is different for each case and have been chosen such that the top of the tubes are at the same height of $z=30$ and $z=25$ for $\alpha_{1}$ and $\alpha_{2}$ cases respectively.

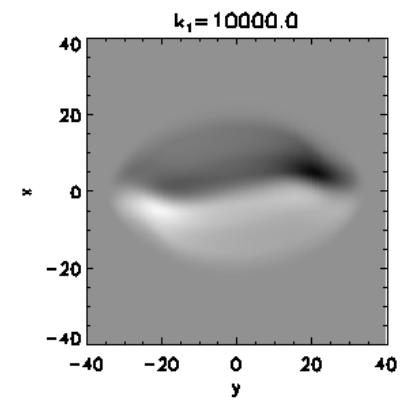

Fig. 14. Synthesised magnetograms at the horizontal plane $z=0$, with maximum absolute scaling $\left|B_{z}\right|=0.5$, for the emerged cases (top row, left to right) $k_{1}=2.5,5.0,10.0,10000.0$ and (bottom row, left to right) $k_{2}=1.0,1.75,2.5$.

slowly from 0.0 and decreases quickly towards this value, the flux tubes have a low tension force.

Although the twist profiles are sufficiently different from each other, our results have shown that there are both similarities and differences in the rise, emergence and expansion stages of the emergence of non-constant twist flux tubes.

The similarities encompass events where, regardless of the twist profile, the low tension cases act similarly and the high tension cases act similarly. During the tubes' rise through the solar interior, we find there is a redistribution of the magnetic field away from the initial perfectly cylindrical structure towards a "magnetically top-heavy" structure, whereby the flux at the rear of the tube reduces but increases in the front portion. This redistribution of field occurs to a greater degree for the lower tension cases due to the inability of a low tension force to maintain the structure of the tube. Although the vertical velocity of the tube is initially well prescribed by the buoyancy force, an imbalance in the radial forces grows and is most exaggerated for the low tension cases due to the reorganisation of the field. Once the tubes surface in the photosphere, a magnetic buoyancy instability will occur if the plasma- $\beta$ falls below 1 . However, the instability does not occur for the low tension cases since they have experienced greater horizontal expansion during their rise to the surface and, therefore, have a weaker magnetic field and higher plasma- $\beta$. Post-instability, the growth rate increases as we move towards higher tension cases and, thus, these tubes rise into the atmosphere quicker. The tubes with the faster growth rate transport more flux into the atmosphere as a function of time and height of the top of the tube, while the tubes with the lower tension force, and longer growth time, experience greater horizontal expansion in the atmosphere.

The differences in the twist profiles arise when the nature of the tension force becomes important as opposed to solely its magnitude. For the $\alpha_{1}$ cases, the non-zero tension force is the same size in the immediate vicinity of the axis and, thus, the tubes experience the same horizontal expansion and decrease in the axial field strength as they rise. However, the tension force increases at varying rates with radius in the $\alpha_{2}$ cases and, therefore, these tubes experience different degrees of expansion and rates of decrease in the axial field strength depending upon their individual tension profiles. All of the cases see an increase in the pitch of the fieldlines but the $\alpha_{1}$ cases, with initially decreasing pitch at outer radii, see the largest increase. At the time of emergence, all of the tubes have non-zero pitch at their front edges. However, the magnitude of the non-zero pitch angle at the front varies in the $\alpha_{1}$ cases and, thus, we see differences in the pitch profiles of the atmospheric field with height, while the $\alpha_{2}$ cases have no significant differences. 
From our results, it appears that the twist profile of the tube bears little significance in determining whether the field will emerge and that the strength of the magnetic field is actually of key importance. The field strength is determined by the degree of horizontal expansion of the tube while it rises through the solar interior and this, in turn, is governed by the height of the axis and the tension profile in the vicinity of the axis. In terms of these quantities, neither profile appears to be significantly better than the other for instigating emergence. Considering the overall profile of the transverse component of the field, $B_{\theta}$, we find that the $k_{1}=1.75$ and $k_{2}=2.5$ cases have approximately the same maximum value for $B_{\theta}$. However, the former case does not emerge while the latter does and this indicates that the location of the maximum $B_{\theta}$ is more important than the actual maximum value. Perhaps, therefore, the amount of flux in the tube is the key factor governing emergence.

In terms of observations, there is very little discernable difference between the pitch angle of the fieldlines once the tension force is reasonably high but the degree of horizontal expansion may give some indication as to the level of twist in the tube. From our results, we find that magnetograms at the solar surface provide the greatest key for distinguishing between the different twist profiles. Future observational studies of emerging active regions will be important in determining whether it is possible to identify such differences in solar magnetograms and, thus, whether twisted emerging flux favours either of the nonconstant twist profiles described in this paper. Additionally, the results from this study and those of Murray et al. (2006) imply that the strength of the field at the time of emergence influences the rate of rise of the flux into the atmosphere. We believe that an observational study of large-scale emerging flux events is required in order to substantiate this theory.

Acknowledgements. Michelle Murray acknowledges financial assistance from the Particle Physics and Astronomy Research Council (PPARC). The computational work for this paper was carried out on the joint STFC and SFC (SRIF) funded linux cluster at the University of St Andrews (Scotland, UK).

\section{References}

Acheson, D. J. 1979, Solar Physics, 62, 23

Archontis, V., Moreno-Insertis, F., Galsgaard, K., Hood, A., \& O'Shea, E. 2004, A\&A, 426, 1047

Archontis, V., Moreno-Insertis, F., Galsgaard, K., \& Hood, A. W. 2005, ApJ, 635,1299

Archontis, V., Galsgaard, K., Moreno-Insertis, F., \& Hood, A. W. 2006, ApJ, $645, \mathrm{~L} 161$

Archontis, V., Hood, A. W., \& Brady, C. 2007, A\&A, 466, 367

Babcock, H. W. 1961, ApJ, 133, 572
Burnette, A. B., Canfield, R. C., \& Pevtsov, A. A. 2004, ApJ, 606, 565 Canfield, R. C., de La Beaujardiere, J.-F., Fan, Y., et al. 1993, ApJ, 411, 362

Emonet, T., \& Moreno-Insertis, F. 1996, ApJ, 458, 783

Emonet, T., \& Moreno-Insertis, F. 1998, ApJ, 492, 804

Fan, Y. 2001, ApJ, 554, L111

Fan, Y. 2005, ApJ, 630, 543

Fan, Y., \& Gibson, S. E. 2003, ApJ, 589, L105

Fan, Y., \& Gibson, S. E. 2004, ApJ, 609, 1123

Frazier, E. N. 1972, Sol. Phys., 26, 130

Galsgaard, K., Moreno-Insertis, F., Archontis, V., \& Hood, A. 2005, ApJ, 618, L153

Galsgaard, K., Archontis, V., Moreno-Insertis, F., \& Hood, A. 2007, ApJ, submitted

Gibson, S. E., \& Fan, Y. 2006, ApJ, 637, L65

Green, L. M., López fuentes, M. C., Mandrini, C. H., et al. 2002, Sol. Phys., 208, 43

Hughes, D. W., Falle, S. A. E. G., \& Joarder, P. 1998, MNRS, 298, 433

Isobe, H., Miyagoshi, T., Shibata, K., \& Yokoyama, T. 2005, Nature, 434, 478

Kaisig, M., Tajima, T., Shibata, K., Nozawa, S., \& Matsumoto, R. 1990, ApJ, 358,698

Kusano, K., Moriyama, K., \& Miyoshi, T. 1998, Phys. Plasmas, 5, 2582

Leake, J. E., \& Arber, T. D. 2006, A\&A, 450, 805

Leka, K. D., Canfield, R. C., McClymont, A. N., \& van Driel-Gesztelyi, L. 1996, ApJ, 462, 547

Lites, B. W., Low, B. C., Martinez Pillet, V., et al. 1995, ApJ, 446, 877

Magara, T. 2001, ApJ, 549, 608

Magara, T. 2004, ApJ, 605, 480

Magara, T. 2006, ApJ, 653, 1499

Magara, T., \& Longcope, D. W. 2001, ApJ, 559, L55

Magara, T., \& Longcope, D. W. 2003, ApJ, 586, 630

Manchester, W., Gombosi, T., DeZeeuw, D., \& Fan, Y. 2004, ApJ, 610, 588

Matsumoto, R., \& Shibata, K. 1992, PASJ, 44, 167

Matsumoto, R., Tajima, T., Shibata, K., \& Kaisig, M. 1993, ApJ, 414, 357

Miyagoshi, T., \& Yokoyama, T. 2003, ApJ, 593, L133

Moreno-Insertis, F., \& Emonet, T. 1996, ApJ, 472, L53

Murray, M. J., \& Hood, A. W. 2007, A\&A, 470, 709

Murray, M. J., Hood, A. W., Moreno-Insertis, F., Galsgaard, K., \& Archontis, V. 2006, A\&A, 460, 909

Newcomb, W. A. 1961, Physics of Fluids, 4, 391

Nordlund, A., \& Galsgaard, K. 1997, A 3D MHD Code for Parallel Computers, Tech. rep., Astronomical Observatory, Copenhagen University

Parker, E. N. 1979, Cosmical magnetic fields: Their origin and their activity (Oxford: Clarendon Press; New York: Oxford University Press), 858

Pevtsov, A. A., Canfield, R. C., \& Metcalf, T. R. 1995, ApJ, 440, L109

Piddington, J. H. 1975, Ap\&SS, 34, 347

Piddington, J. H. 1981, Ap\&SS, 75, 273

Roberts, B. 1991, in Adv Sol. Sys. Magnetohydrodynamics, ed. E. R. Priest, \& A. W. Hood, 105

Schmieder, B., Demoulin, P., Aulanier, G., \& Golub, L. 1996, ApJ, 467, 881

Shibata, K., Tajima, T., Matsumoto, R., et al. 1989a, ApJ, 338, 471

Shibata, K., Tajima, T., Steinolfson, R. S., \& Matsumoto, R. 1989b, ApJ, 345, 584

Shibata, K., Nozawa, S., \& Matsumoto, R. 1992, PASJ, 44, 265

Solanki, S. K. 2003, A\&AR, 11, 153

Thomas, J. H., \& Nye, A. H. 1975, Phys. Fluids, 18, 490

Yokoyama, T., \& Shibata, K. 1995, Nature, 375, 42

Yu, C. P. 1965, Phys. Fluids, 8, 650

Zwaan, C. 1985, Sol. Phys., 100, 397 